\section{Update on orbital anatomy}

\begin{abstract}
The orbit is a confined space bounded by four bony walls. Apart from the globe, orbital fat, and extraocular muscles it contains many important neurovascular structures. It is intimately related to the paranasal sinuses as well as the anterior and middle cranial fossae. Consequently, sinus pathology and intracranial disease may spread to involve the orbit and the converse applies. A thorough understanding of orbital anatomy is essential to fully appreciate the effects of disease on the orbit and is of paramount importance for performing safe orbital surgery. The anatomy of the orbit is discussed with reference to relevant orbital disease.

Eye (2006) 20, 1119-1129. doi:10.1038/sj.eye.6702376
\end{abstract}

Keywords: orbit; anatomy; orbital diseases

\section{The bony orbit}

The orbit is a bony pyramid with four walls: a roof, lateral wall, floor, and medial wall. The base of the pyramid is the orbital entrance, which is roughly rectangular (Figure 1). It measures $4 \mathrm{~cm}$ wide by $3.5 \mathrm{~cm}$ high and is rotated laterally. ${ }^{1}$ Because of this lateral rotation, the lateral orbital rim is approximately at the equator of the globe, making the globe relatively exposed laterally. The geometry of the orbit is such that it expands to its maximum dimension $1 \mathrm{~cm}$ behind the rim. The apex of the orbital pyramid is situated $44-50 \mathrm{~mm}$ posteriorly and contains important neurovascular structures crammed into a very tight space. The medial orbital walls are parallel, approximately $2.5 \mathrm{~cm}$ apart and separated by paired ethmoid sinuses. Each lateral wall forms a $45^{\circ}$ angle with its respective medial wall, resulting in $90^{\circ}$ between the two lateral walls (Figure 2). The orbital volume is roughly $30 \mathrm{ml}$, of which $7 \mathrm{ml}$ is occupied by the globe. There is some variation with sex and race. ${ }^{2}$
C René

\section{Medial wall}

The medial wall (Figure 3) is roughly rectangular and extends from the anterior lacrimal crest (frontal process of the maxilla) to the orbital apex. At its anterior aspect, it contains the lacrimal fossa which is bounded by the anterior and posterior lacrimal crests. The latter is a well-defined ridge on the thin lacrimal bone, which forms part of the lateral wall of the nose and overlies the root of the middle turbinate. The vast majority of the medial wall is comprised of the lamina papyracea. This paperthin bone overlying the ethmoid sinus facilitates the spread of infection, in cases of ethmoid sinusitis, into the orbit with subperiosteal abscess formation and/or orbital cellulitis (Figure 4). The lamina papyracea fractures readily follow blunt orbital trauma and is also readily breached in the course of ethmoid sinus surgery, exposing the orbit to the risk of inadvertent surgical injury. ${ }^{3}$ The thick bone of the sphenoid body forms the most posterior part of the medial wall, adjoining the optic canal. The medial wall articulates with the roof at the fronto-ethmoid suture, which marks the upper limit of safe bone removal during medial wall decompression. Removal of bone above this suture carries the risk of cerebrospinal fluid leakage owing to exposure of the dura of the frontal lobe. The anterior and posterior ethmoidal nerves and vessels leave the orbit via their respective foramina located in the frontoethmoid suture and the vessels should be clipped or cauterized to avoid potentially devastating orbital haemorrhage in the course of extraperiosteal medial wall dissection. The medial wall articulates with the floor at the maxillo-ethmoid suture, which marks the position of the thick inferomedial bony strut which is usually left intact anteriorly to support the globe and prevent hypoglobus following orbital decompression.

\section{Floor}

The floor (Figure 5) is triangular and slopes gradually upwards to merge almost
Department of

Ophthalmology, Addenbrooke's Hospital, Hills Road, Cambridge CB2 2QQ, UK

Correspondence: C René, Tel: + 01223216500 ; Fax: + 01223217968 E-mail: cornelius.rene@ addenbrookes.nhs.uk

Received: 8 March 2006 Accepted: 22 March 2006 

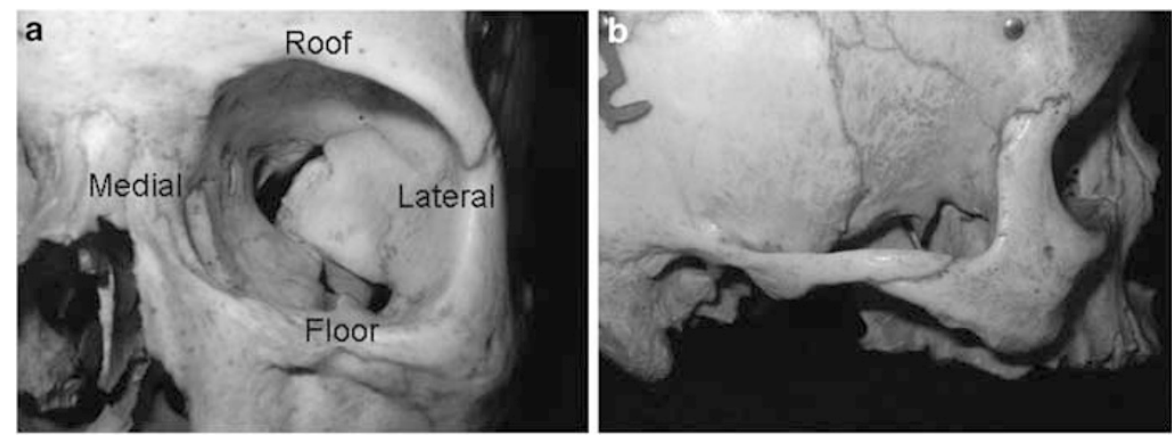

Figure 1 (a) The bony orbit is pyramidal shape of the orbit. The base is situated anteriorly and the apex posteriorly. (b) The orbital entrance is rotated laterally.

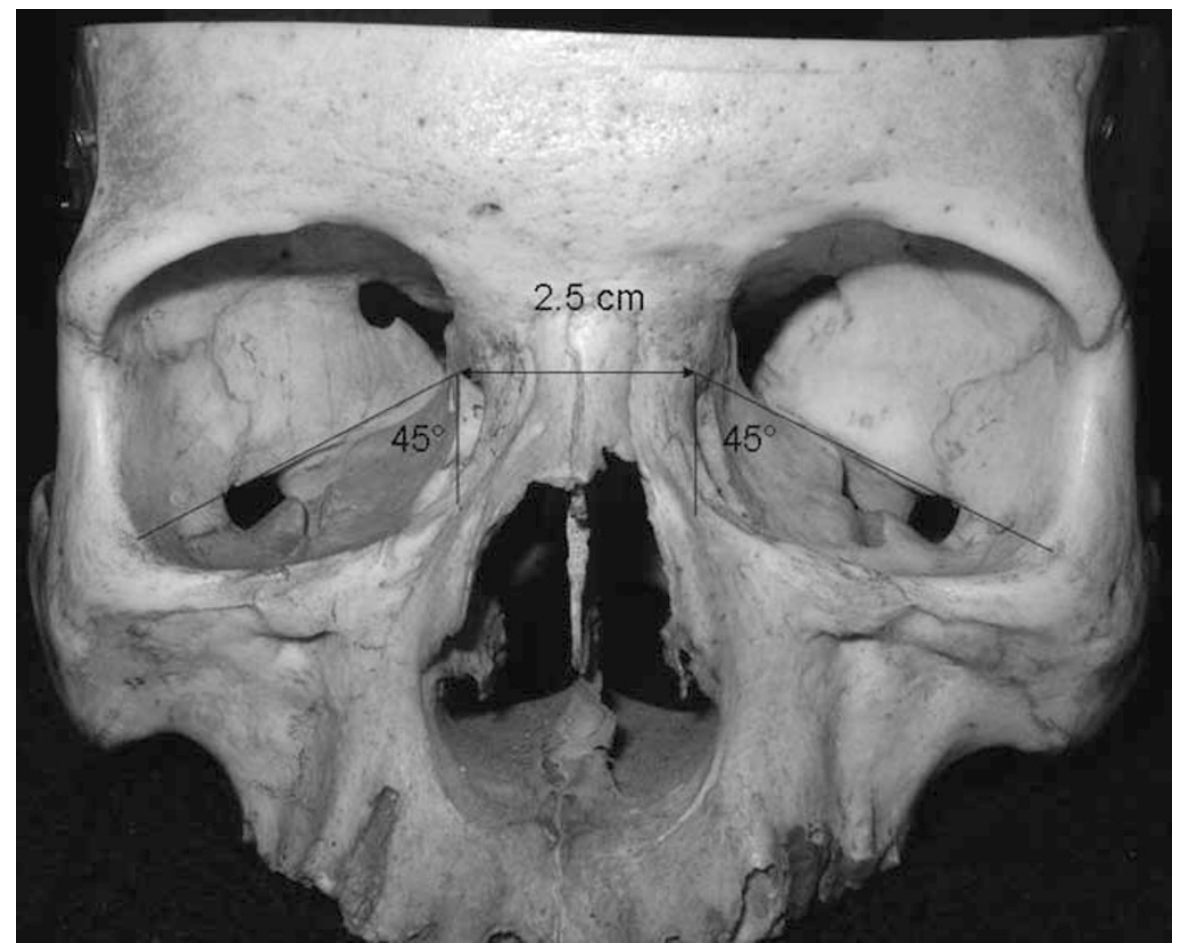

Figure 2 The relationship between the medial walls, the paired ethmoid sinuses, and the corresponding lateral walls.

imperceptibly with the medial wall at the

maxilloethmoid suture. It is formed mainly by the orbital plate of the maxilla with contributions from the zygoma anterolaterally and the palatine bone at its most posterior limit. The orbital floor is the roof of the maxillary sinus. In its posterolateral two-thirds, the floor is separated from the lateral wall by the inferior orbital fissure through which the maxillary division of the trigeminal nerve $\left(V_{2}\right)$ enters the orbit, having traversed the pterygopalatine fossa from the foramen rotundum. The nerve lies exposed in a sulcus in the posterior part of the floor where it is prone to surgical injury. It passes forward into the infraorbital canal to exit the orbit at the infraorbital foramen. Medial to the infraorbital nerve, the orbital floor is relatively thin and fractures easily. This is the portion of the floor which is usually removed during floor decompression. Other structures crossing the inferior orbital fissure include the infraorbital branch of the maxillary artery, the inferior ophthalmic vein, and autonomic branches of the pterygopalatine ganglion. The anterior part of the inferior orbital fissure contains no important structures.

\section{Lateral wall}

The lateral wall (Figures 6 and 7), the thickest of the orbital walls, is formed by the greater wing of sphenoid posteriorly and the zygoma anteriorly. It is separated 


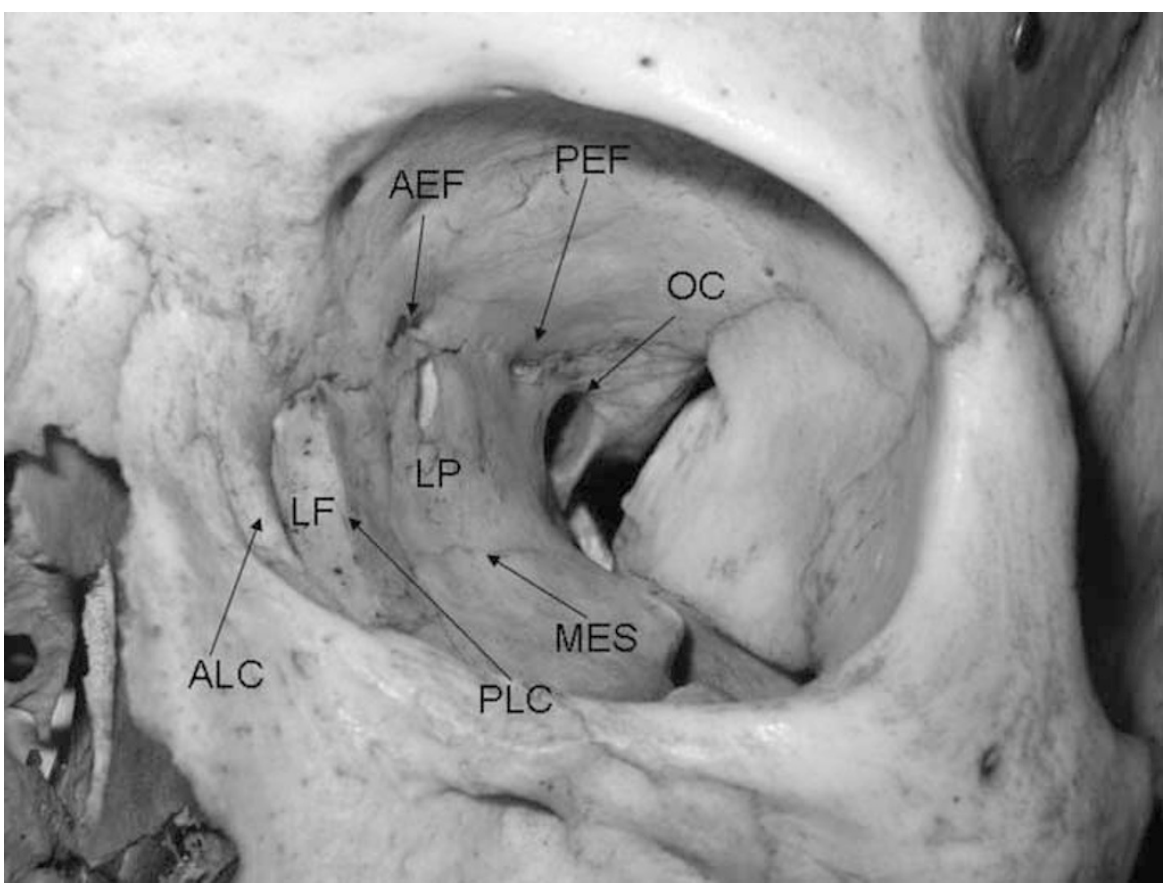

Figure 3 The anatomy of the medial orbital wall. Key: ALC, anterior lacrimal crest; LF, lacrimal fossa; PLC, posterior lacrimal crest; LP, lamina papyracea; AEF, anterior ethmoidal foramen; PLF, posterior ethmoidal foramen; OC, optic canal; MES, maxilloethmoid suture.

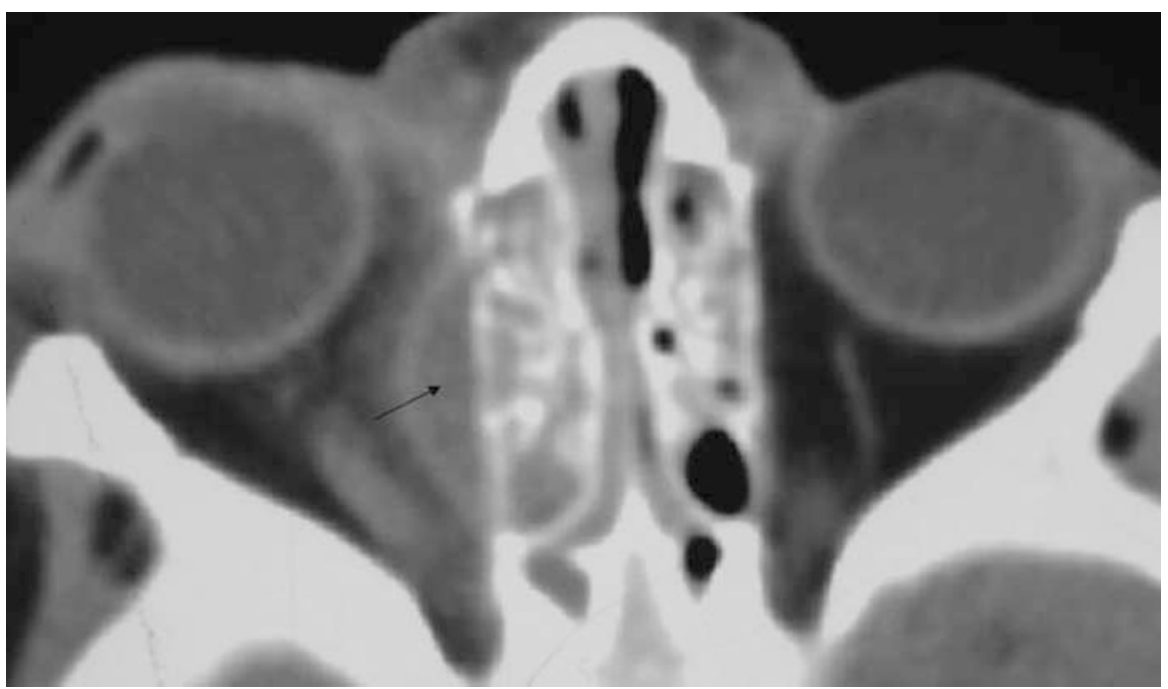

Figure 4 Axial computed tomography scan of orbits revealing right subperiosteal orbital abscess (arrow) secondary to ethmoid sinus disease in a child.

from the floor by the inferior orbital fissure and from the roof by the superior orbital fissure (posteriorly) and the frontosphenoid suture. One or more recurrent meningeal branches of the ophthalmic artery (internal carotid supply) exit the orbit via the frontosphenoid suture to anastomose with the middle meningeal artery (external carotid supply). In its posterior part, the greater wing of sphenoid is interposed between the middle cranial fossa and the orbit. Congenital absence of the greater wing of sphenoid in neurofibromatosis may result in pulsatile proptosis owing to orbital encephalocoele. ${ }^{4,5}$ The lateral canthal tendon, Whitnall's ligament, the lateral horn of the levator, Lockwood's ligament, and the check ligament of lateral rectus all attach at the lateral tubercle, found $3-4 \mathrm{~mm}$ behind the lateral orbital rim. The zygomaticofacial and zygomaticotemporal 

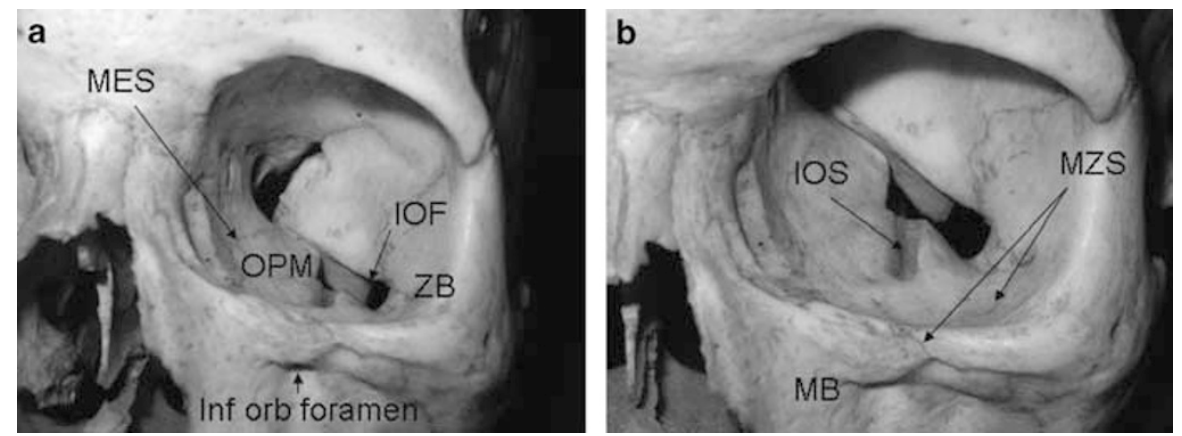

Figure 5 Anatomy of the orbital floor, anterior view (a) and slightly from above (b). Note gradual upward inclination to merge with the medial wall. Key: OPM, orbital plate of maxilla; MB, maxillary bone; IOF, inferior orbital fissure; Inf orb foramen, infraorbital foramen; IOS, infraorbital sulcus; MZS, maxillozygomatic suture; MES, maxilloethmoid suture; ZB, zygomatic bone.

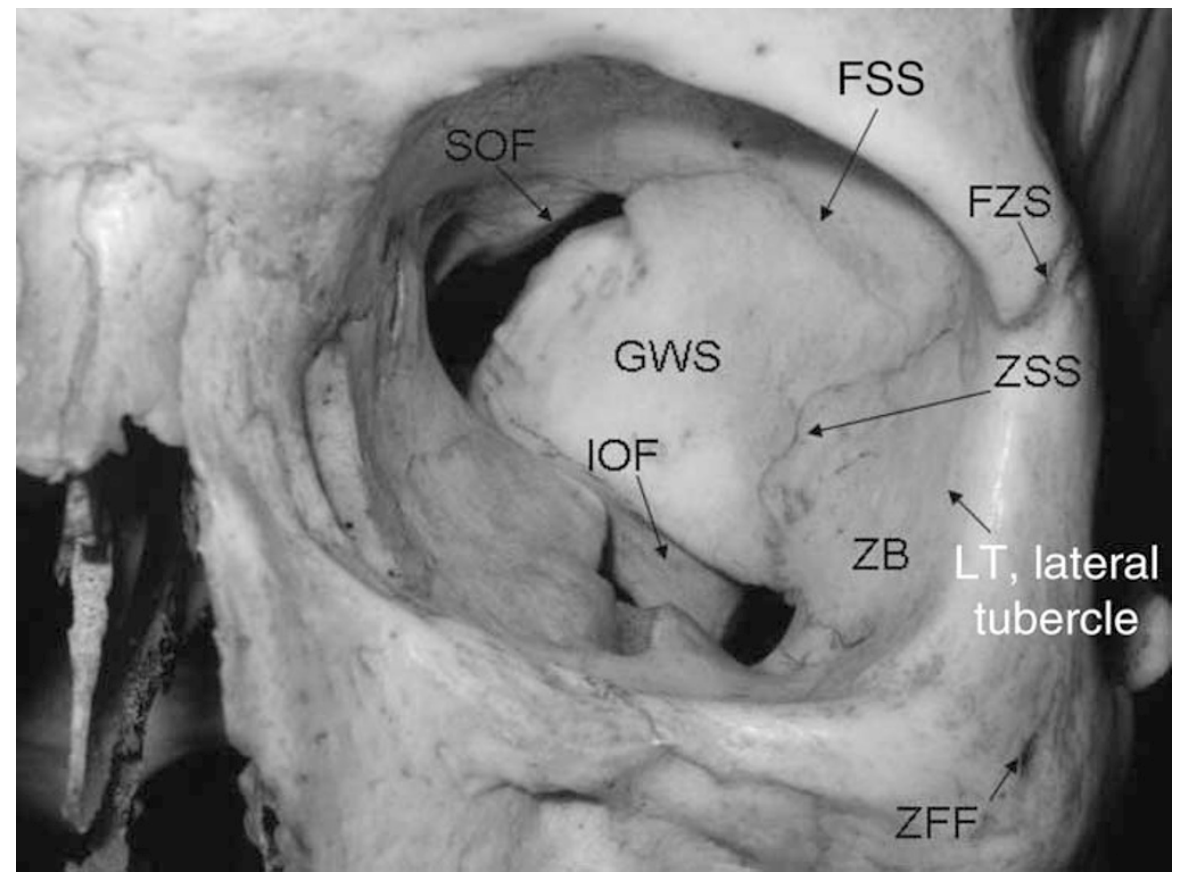

Figure 6 Anatomy of the lateral orbital wall, anterior view. Key: GWS, greater wing of sphenoid; SOF, superior orbital fissure; FZS, frontozygomatic suture; FSS, frontosphenoid suture; ZSS, zygomaticosphenoid suture; ZB, zygomatic bone; ZFF, zygomaticofacial oramen; LT, lateral tubercle.

neurovascular structures leave the orbit via their respective foramina on the lateral wall. During lateral orbitotomy surgery, the superior bone cut is usually made just above the frontozygomatic suture, at which point the middle cranial fossa is in close proximity. ${ }^{6}$ The lateral wall removal is completed by fracturing the bone at the zygomaticosphenoid suture.

\section{Orbital roof}

The orbital roof (Figure 8) is composed almost entirely of the frontal bone with a tiny contribution from the lesser wing of sphenoid tapering posteriorly into the anterior clinoid process. It has a ridged, convex upper surface which forms the floor of the anterior cranial fossa. The paired roofs dip medially towards the cribriform plate. The orbital roof is very strong and rarely fractures. There is variable pneumatization of the roof by the frontal sinus. Just posterior to the rim at the anteromedial aspect of the roof is a small depression housing the trochlea, which can be safely lifted off its fossa in extraperiosteal dissection of the roof. However, care is required to avoid damage to the trochlea and scarring in this region results in a Brown's syndrome. At the junction of the medial third and lateral two-thirds of the superior orbital rim, the supraorbital notch (foramen in $25 \%$ of people) 


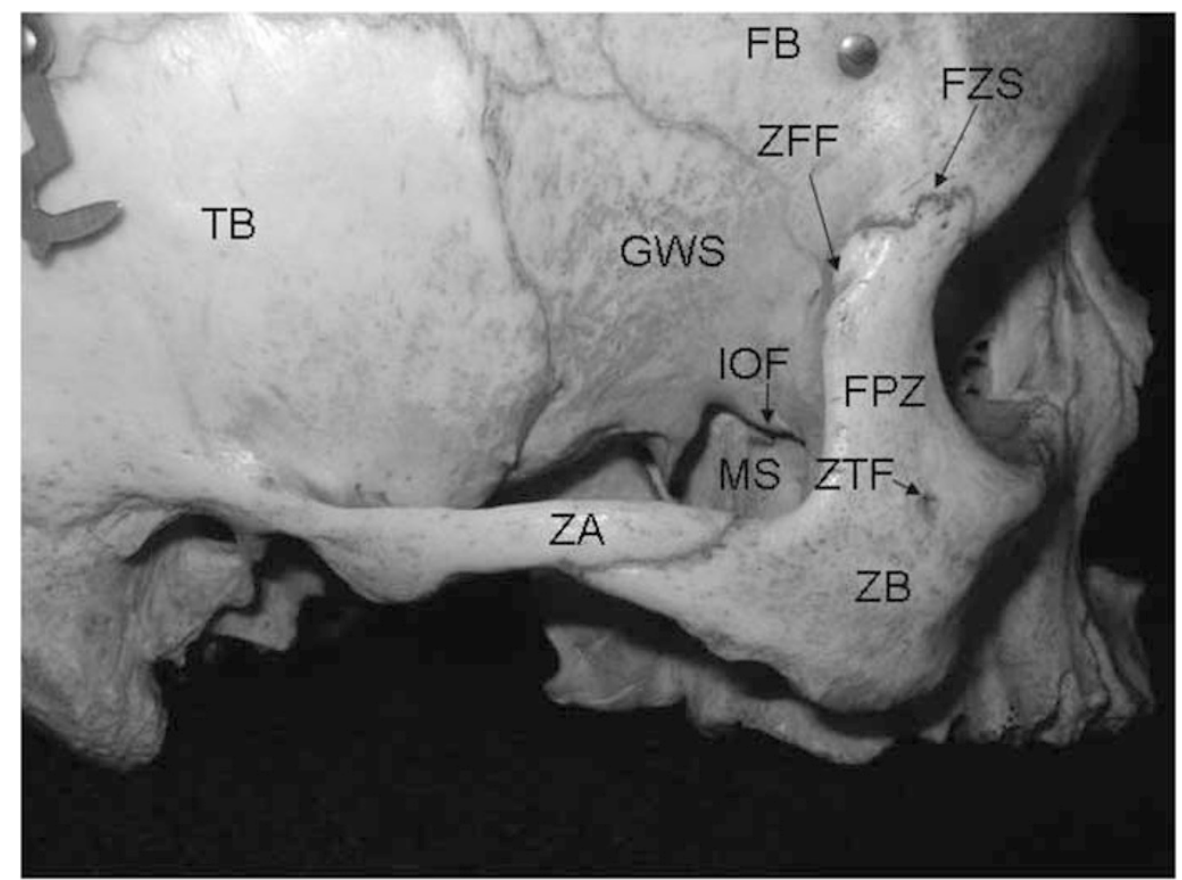

Figure 7 Lateral view of the lateral orbital wall and its relationships. Key: FZS, frontozygomatic suture; FPZ, frontal process of the zygoma; ZB, zygomatic bone; ZA, zygomatic arch; ZFF, zygomaticofacial foramen; ZTF, zygomaticotemporal foramen; IOF, inferior orbital fissure; MS, maxillary sinus; TB, temporal bone; FB, frontal bone; GWS, greater wing of sphenoid.
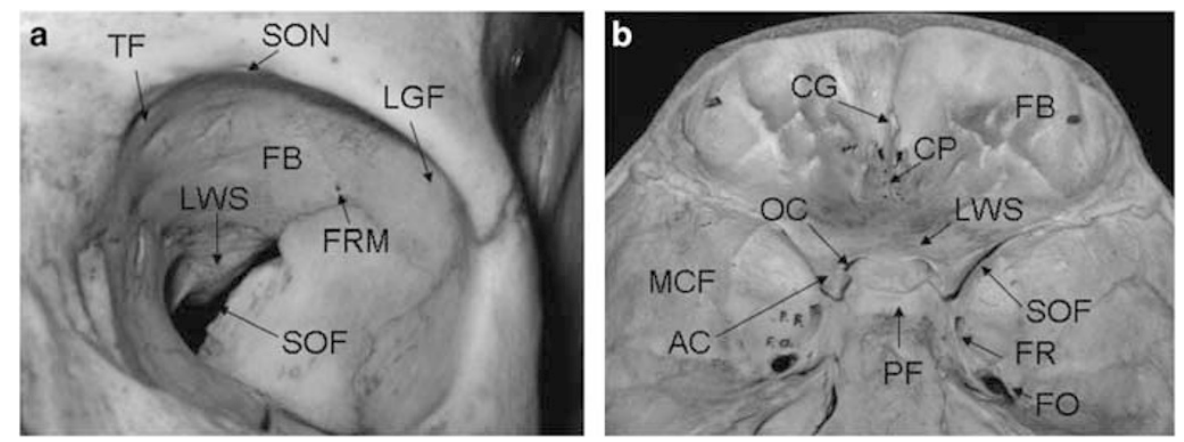

Figure 8 Orbital roof viewed from the orbit (a) and from the cranial aspect (b) with dorsum sellae removed. Key: SON, supraorbital notch; LF, lacrimal gland fossa; TF, trochlear fossa; SOF, superior orbital fissure; LWS, lesser wing of sphenoid; FB, frontal bone; CG, crista galli; $\mathrm{CP}$, cribriform plate; $\mathrm{OC}$, optic canal; FR, foramen rotundum; FO, foramen ovale; $\mathrm{MCF}$, middle cranial fossa; $\mathrm{PF}$, pituitary fossa; AC, anterior clinoid process; FRM, foramen for recurrent meningeal artery

transmits the supraorbital neurovascular bundle and is almost directly above its inferior counterpart. The lacrimal gland is housed in a shallow fossa in the anterolateral aspect of the roof.

\section{The infratemporal and pterygopalatine fossae}

Deep to the zygomatic arch is the infratemporal fossa (Figure 9), which contains the terminal fibres of the temporalis muscle, the maxillary artery, the maxillary vein, the buccal fat pad, and the temporalis fat pads. This space communicates with the orbit anteriorly via the anterior part of the inferior orbital fissure, thereby providing a route by which blood from the temporal fossa can accumulate in the orbit. In a deeper location still lies the pterygopalatine fossa, which communicates with the posterior part of the inferior orbital fissure. The pterygopalatine fossa is occupied by the pterygopalatine ganglion and is traversed by the maxillary nerve, on its way from the foramen rotundum to the inferior orbital groove, and the infraorbital branch of the maxillary artery. 


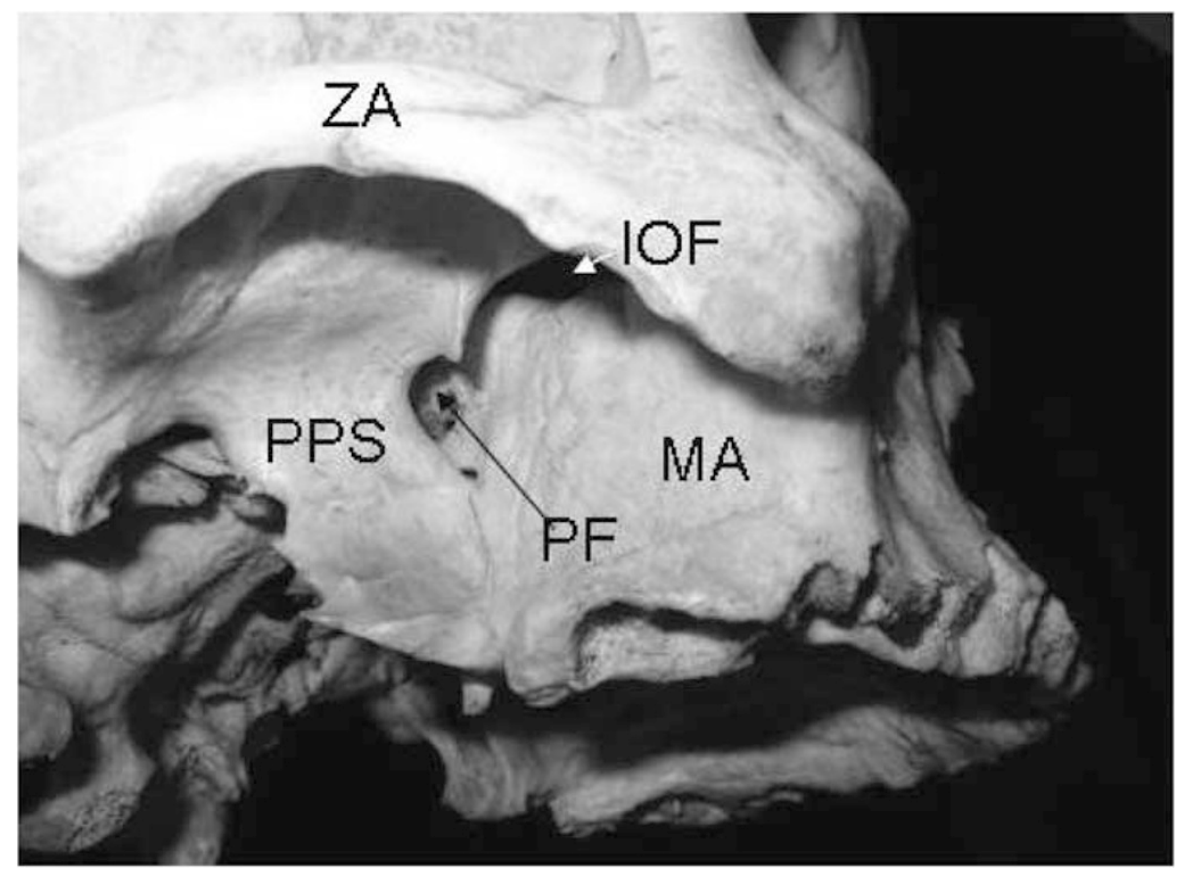

Figure 9 Anatomy and relationships of the infratemporal and pterygopalatine fossae. Key: ZA, zygomatic arch; PF, pterygopalatine fossa; IOF, inferior orbital fissure; MA, maxillary antrum; PPS, pterygoid process of the sphenoid bone.

\section{The orbital apex}

The orbital apex provides the route of communication between the intracranial cavity and the orbit via the superior orbital fissure and the optic foramen. The posterior part of the inferior orbital fissure and its connections with the pterygopalatine ganglion also occupies the orbital apex. The high concentration of important neurovascular structures in this cramped space accounts for the orbital apex syndrome in which multiple cranial nerve palsies accompany loss of vision and a relative afferent pupil defect in certain inflammatory syndromes (e.g. Tolosa-Hunt) and apical tumours, even in the absence of significant inflammatory signs or mass effect. ${ }^{7}$

\section{The optic canal}

The optic canal is housed in the lesser wing of the sphenoid. Its entrance, the optic foramen, occupies the most superior and medial part of the orbit. When viewed from the orbit it has a $45^{\circ}$ medial and $15^{\circ}$ upward angulation. Both canals are symmetrical with a circumference of 6 and $8 \mathrm{~mm}$ length. Pathology, such as optic nerve glioma, leads to an enlargement of the optic canal with resultant asymmetry.

The optic canal has three bony walls: a medial wall, lateral wall, and roof. An impression on the body of the sphenoid forms the medial wall, but there are variations in this region and in up to $42 \%$ of cases posterior ethmoid air cells (Onodi cells) form the medial wall of the canal. ${ }^{8,9}$ In such cases, the optic nerve is at risk of damage in ethmoid surgery. The root of the lesser wing of sphenoid forms the roof of the canal and the lateral wall is formed by the bony strut that spans from the body of the sphenoid to the anterior clinoid process. The intracanalicular part of the optic nerve is susceptible to damage within the optic canal. ${ }^{10,11}$

\section{The superior orbital fissure}

The superior orbital fissure lies between the greater and lesser wings of sphenoid and contains many critical neurovascular structures (Figure 10). It usually narrows superotemporally and widens medially, below the optic foramen. The annulus of Zinn, a tight fibrous ring divides the superior orbital fissure into intraconal and extraconal spaces.

The four rectus muscles arise from the annulus of Zinn. The superior and medial recti arise from the part of the annulus attached to the body of the sphenoid, adjacent to the optic foramen. A tendinous portion of the annulus spanning from the body of sphenoid to the greater wing gives rise to the inferior rectus. Lateral rectus arises from the body of the greater wing along the lateral border of the superior orbital fissure and a separate tendinous part of the annulus that spans from 


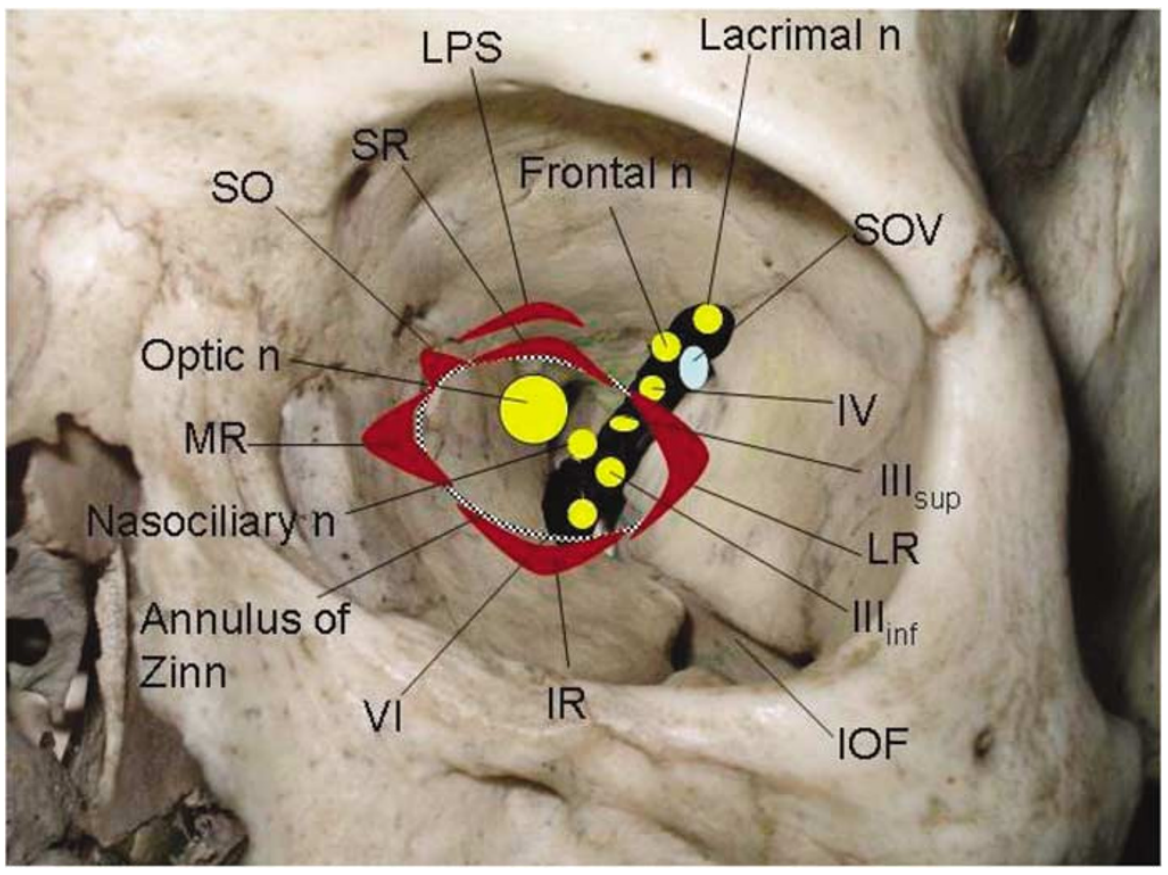

Figure 10 Anatomy of the left orbital apex, highlighting the extraocular muscle origins and the contents of the superior orbital fissure. Key: LPS, levator muscle; SR, superior rectus; LR, lateral rectus; IR, inferior rectus; MR, medial rectus; SO, superior oblique; $\mathrm{SOV}$, superior ophthalmic vein; $\mathrm{III}_{\text {sup }}$, superior division of oculomotor nerve; $\mathrm{III}_{\mathrm{inf}}$, inferior division of oculomotor nerve; IOF, inferior orbital fissure.

the greater wing to lesser wing of sphenoid across the superior orbital fissure and lateral to the optic nerve.

The ophthalmic division of the trigeminal nerve $\left(\mathrm{V}_{1}\right)$ divides in the cavernous sinus into lacrimal, frontal, and nasociliary branches. The lacrimal and frontal nerves are uppermost in the superior orbital fissure, outside the annulus of Zinn. Just below them is the superior ophthalmic vein. The trochlear nerve (IV) enters the orbit just outside the annulus of Zinn and closely applied to its superior fibres. The remaining structures enter the orbital apex inside the annulus of Zinn, within the intraconal space. They include the optic nerve, superior and inferior divisions of the oculomotor nerve (III), the nasociliary branch of $\mathrm{V}_{1}$, the abducens nerve (VI), and sympathetic nerve fibres.

\section{The extraocular muscles and their nerve supply}

The rectus muscles originate from the annulus of Zinn as described above and course forward through the orbital fat to insert onto the globe in their respective meridia along the spiral of Tillaux 5-8 mm from the limbus, medial rectus being closest to the limbus and superior rectus furthest from it. Together with the intramuscular septa they demarcate the intraconal space.

The medial rectus is closely applied to the lamina papyracea of the medial wall and is prone to entrapment in medial wall fractures with resultant retraction of the globe and narrowing of the palpebral aperture on attempted abduction (pseudo-Duanes syndrome). ${ }^{12}$ It is also susceptible to iatrogenic damage in ethmoid sinus surgery, when inadvertent orbital entry may occur. ${ }^{3}$

The inferior rectus lies close to the floor posteriorly but has fat interposed anteriorly. In blow-out floor fractures, the inferior rectus may prolapse into the bony defect together with fat herniating into the maxillary antrum. Incarceration of the inferior rectus muscle sheath and its fibrous septa that radiate towards the orbital floor may occur in blow-out floor fractures, even in the absence of a significant bony defect or soft tissue herniation, resulting in restricted elevation and depression of the eye.

The lateral rectus muscle is separated from the lateral orbital wall by fat. Posteriorly, the ciliary ganglion lies between it and the optic nerve. The nerves entering the orbit within the annulus of Zinn also separate it from the optic nerve. The lacrimal gland lies superior to its terminal portion and the lacrimal artery and nerve also lie above it.

The levator palpebrae superioris originates from the lesser wing of sphenoid just above and slightly medial to the superior rectus. The two arise from the same embryonic mesenchyme and are intimately related. They diverge about the equator of the globe, at which point the superior rectus travels inferiorly to attach to the globe 
whereas the levator continues forward becoming aponeurotic in the region of Whitnall's ligament. The aponeurosis fans out to insert onto the anterior surface of the tarsal plate and the eyelid skin. It also attaches via the 'horns' adjacent to the medial and lateral canthal tendons. While passing forward in the orbit, the nasociliary nerve and ophthalmic artery cross from lateral to medial below the superior rectus. Superior relations of the levator are the supraorbital artery and the lacrimal and frontal nerves.

The superior oblique arises from the annulus of Zinn superomedially and courses close to the periorbita just above the fronto-ethmoid suture towards the trochlea. After passing through the trochlea, its tendon fans out laterally, inferiorly, and posteriorly below the superior rectus to insert onto the globe in a broad fashion extending to the posterolateral aspect of the globe.

The inferior oblique arises from the periorbita of the inferomedial orbit just lateral to the origin of the nasolacrimal duct and courses laterally and posteriorly below the inferior rectus. Before inserting onto the inferolateral aspect of the globe in a broad attachment, the muscle is enveloped by fibres of the capsulopalpebral fascia and Lockwood's inferior suspensory ligament of the globe.

The extraocular muscles are supplied by the oculomotor nerve (III) except lateral rectus, which is supplied by the abducens nerve (VI) and the superior oblique, which is supplied by the trochlear nerve (IV). The nerve supply to the recti travel forward in the intraconal space and enter the respective muscles on their deep surface at the junction of the posterior third and anterior two-thirds.

The oculomotor nerve divides in the cavernous sinus into superior and inferior divisions that enter the orbit separately inside the annulus of Zinn. The superior division supplies the levator and superior rectus muscles, whereas the inferior division supplies the medial rectus, inferior rectus and the inferior oblique. The branch to inferior oblique travels along the lateral border of inferior rectus and enters the inferior oblique as it crosses the inferior rectus, where it is susceptible to iatrogenic injury. It carries preganglionic parasympathetic fibres to the ciliary body and iris sphincter which synapse in the ciliary ganglion.

The trohlear nerve enters the orbit just outside the annulus, having crossed superior to the oculomotor nerve in the lateral wall of the cavernous sinus. It travels forward in the orbit crossing from lateral to medial to enter the lateral border of superior oblique at the junction of the posterior third and anterior two-thirds.

The abducens nerve enters the orbit within the cone and travels along the medial surface of lateral rectus piercing the muscle at the junction of the posterior third and anterior two-thirds.

\section{Sensory nerves}

The sensory nerves of the orbit (Figure 11) derive mostly from the ophthalmic division of the trigeminal nerve $\left(\mathrm{V}_{1}\right)$ with some contribution from the maxillary division $\left(\mathrm{V}_{2}\right)$. $\mathrm{V}_{1}$ divides in the lateral wall of the cavernous sinus into lacrimal, frontal, and nasociliary branches that enter the superior orbital fissure.

The lacrimal and frontal nerves enter the fissure outside the annulus of Zinn and travel forward in the superior orbit. The lacrimal nerve, the smallest branch of $\mathrm{V}_{1}$, travels along the superior border of the lateral rectus muscle towards the lacrimal fossa, where it supplies postganglionic secretomotor fibres to the lacrimal gland and sensory fibres to the surrounding skin and conjunctiva. The preganglionic secretomotor fibres originate in the facial nerve nucleus in the brainstem and travel in the nervus intermedius to enter the internal auditory meatus. They pass through the geniculate ganglion to reach the pterygopalatine ganglion via the greater petrosal nerve. After synapsing in the pterygopalatine ganglion, the postganglionic secretomotor fibres join the maxillary nerve which they later leave, via its zygomaticotemporal branch, to anastomose with the lacrimal nerve just before it supplies the lacrimal gland.

The frontal nerve, the largest branch of $\mathrm{V}_{1}$, passes anteriorly in the superior orbit between the levator muscle and the periorbita. It divides into the supraorbital and supratrochlear branches. The former exits at the supraorbital notch and occupies a bony canal in 25\% of cases. It supplies the brow, forehead, and scalp just beyond the vertex. The supratrochlear nerve passes medially towards the trochlea and supplies the medial upper lid and forehead.

The nasociliary nerve enters the superior orbital fissure within the muscle cone, crosses over the optic nerve and passes forward between the superior oblique and medial rectus muscles. Its branches include the anterior and posterior ethmoidal nerves, two or three long posterior ciliary nerves to the globe and the sensory root to the ciliary ganglion. It terminates as the infratrochlear nerve which supplies the medial canthus and the tip of the nose.

The maxillary nerve $\left(V_{2}\right)$ enters the pterygopalatine fossa from the foramen rotundum. After giving off sphenopalatine, posterior superior alveolar, and zygomatic branches the main bulk of the nerve passes through the inferior orbital fissure to enter the infraorbital sulcus as the infraorbital nerve, which passes forward through the infraorbital canal exiting at the infraorbital foramen and supplying the lower lid skin and conjunctiva, cheek, and the upper lip. Within the infraorbital canal, the infraorbital nerve gives off the anterior superior alveolar branch supplying the upper front teeth. The zygomatic branch of $\mathrm{V}_{2}$ passes through 


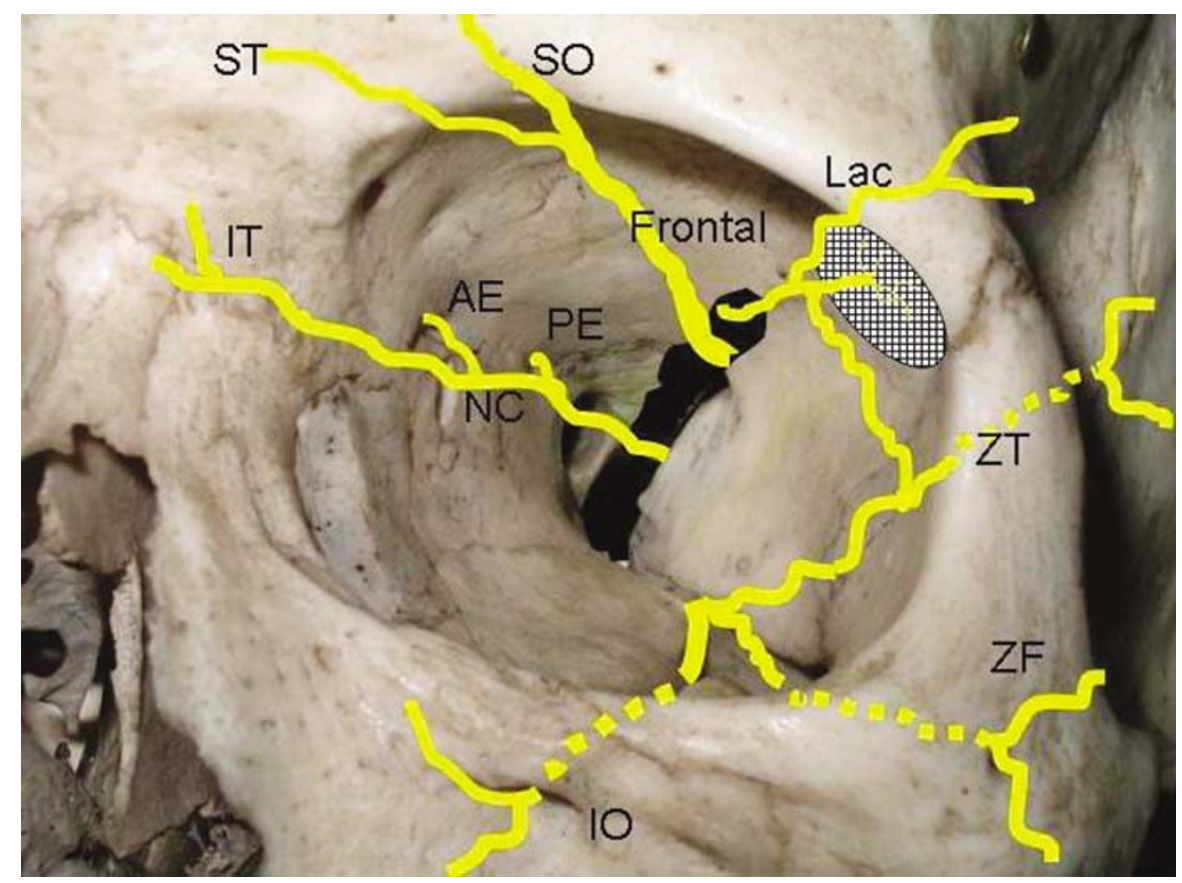

Figure 11 Sensory nerve supply to the orbit. Note that the long posterior ciliary nerves and the sensory root to the ciliary ganglion are not depicted. The hatched structure is the lacrimal gland. Key: ST, supratrochlear nerve; SO, supraorbital nerve; Lac, lacrimal nerve; $\mathrm{ZT}$, zygomaticotemporal nerve; $\mathrm{ZF}$, zygomaticofacial nerve; $\mathrm{IO}$, infraorbital nerve; $\mathrm{NC}$, nasociliary nerve; $\mathrm{AE}$, anterior ethmoidal nerve; $\mathrm{PE}$, posterior ethmoidal nerve; IT, infratrochlear nerve.

the inferior orbital fissure and divides into zygomaticotemporal and zygomaticofacial branches that supply the skin overlying the lateral orbit and zygoma. The zygomaticotemporal branch also delivers secretomotor fibres destined for the lacrimal gland to the lacrimal nerve.

\section{The ciliary ganglion}

The ciliary ganglion sits on the lateral aspect of the optic nerve, close to the orbital apex. It receives a sympathetic, parasympathetic, and sensory root. The sympathetic fibres, destined for the iris dilator and ocular blood vessels, reach the ganglion from the carotid plexus via the superior orbital fissure. Preganglionic parasympathetic fibres to the iris sphincter and ciliary body are delivered to the ganglion by the nerve to inferior oblique. The sensory fibres to the globe are supplied by the nasociliary nerve. Only the parasympathetic fibres synapse in the ciliary ganglion. Five to six short ciliary nerves pass from the ciliary ganglion to the globe, inserting around the optic nerve.

\section{The arterial supply of the orbit}

The ophthalmic artery provides the main arterial supply of the orbit with contributions from the maxillary and middle meningeal arteries, which are branches of the external carotid artery (Figure 12). There are significant anastomoses between the internal and external carotid circulations.

We owe much to Hayreh ${ }^{13,14}$ and Hayreh and Dass ${ }^{15,16}$ for our understanding of the anatomy of the ophthalmic artery. Its origin is variable as are its orbital branches and the following description is of the usual course and branching pattern. The ophthalmic artery arises from the internal carotid artery just after it leaves the cavernous sinus and traverses the optic canal on the inferolateral aspect of the optic nerve within the dural sheath to enter the orbit. The artery crosses from lateral to medial above the optic nerve in about $80 \%$ of cases and below the nerve in about $20 \%$. The central retinal artery arises near the orbital apex and enters the optic nerve on its ventral aspect, about $1 \mathrm{~cm}$ behind the globe, to occupy a central position within the optic nerve. Two or three posterior ciliary arteries subdivide into about 15 short posterior ciliary arteries, that supply the optic nerve head and choroid, and two long posterior ciliary arteries which supply the ciliary body and iris. The lacrimal artery travels superolaterally along the upper border of the lateral rectus to supply the lacrimal gland. It gives off zygomatic and lateral palpebral branches and anastomoses with the middle meningeal artery via the recurrent meningeal artery. The posterior and anterior ethmoidal arteries enter their respective foramina. The 


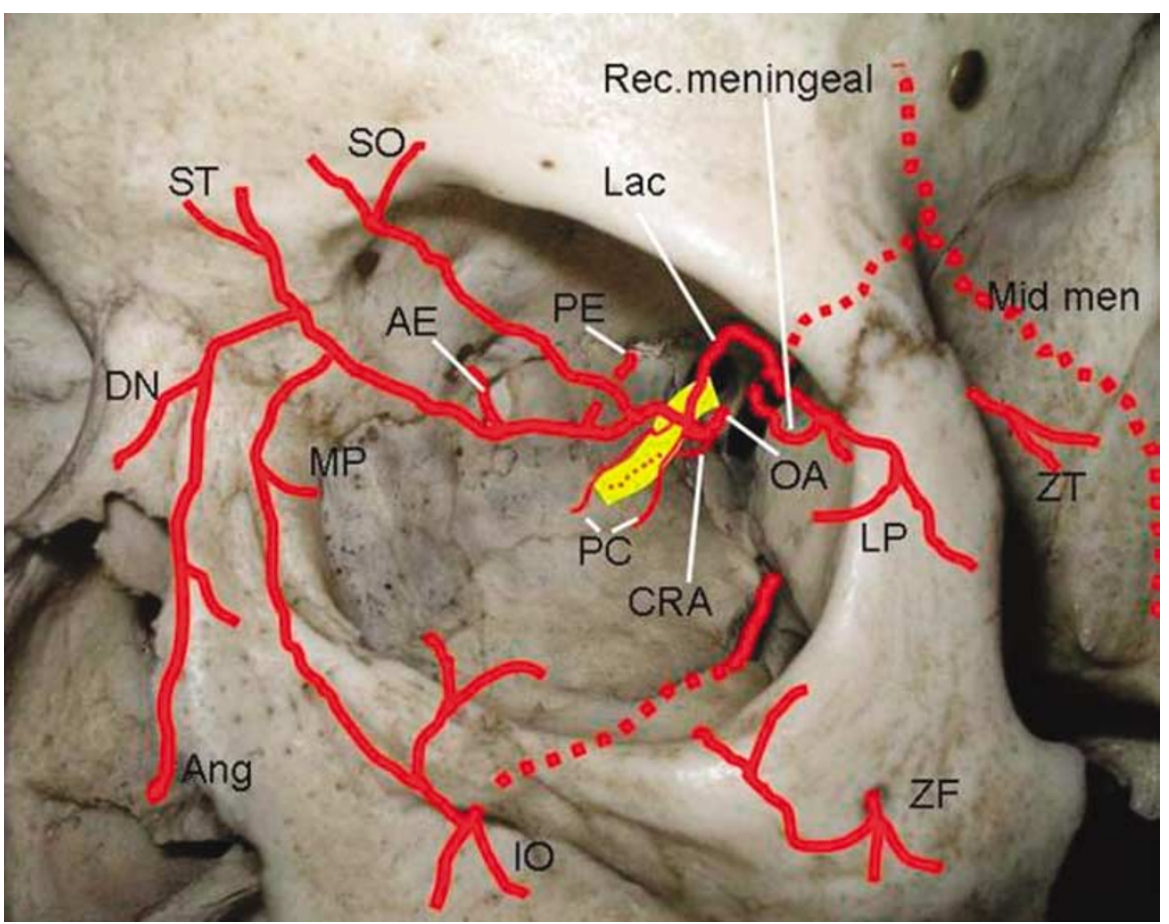

Figure 12 Arterial supply to the orbit. Note the anastomoses between external and internal carotid supplies. The muscular branches, short posterior ciliary, long posterior ciliary, and anterior ciliary arteries are not depicted. The yellow structure is the optic nerve. Key: OA, ophthalmic artery; CRA, central retinal artery; PC, posterior ciliary artery; Lac, lacrimal artery; LP, lateral palpebral artery; IO, infraorbital artery; ZT, zygomaticotemporal artery; ZF, zygomaticofacial artery; Mid men, middle meningeal artery; Rec meningeal, recurrent meningeal artery; Ang, angular artery; MP, medial palpebral artery; DN, dorsal nasal artery; ST, supratrochlear artery; SO, supraorbital artery; $\mathrm{AE}$, anterior ethmoidal artery; PE, posterior ethmoidal artery.

muscular branches supply the extraocular muscles. Within the rectus muscles the muscular arteries divide into two anterior ciliary arteries, except lateral rectus that contains only one, which pierce the globe at the tendinous insertion to anastomose with the long posterior ciliary arteries and supply the anterior segment structures. The supraorbital artery travels forward in the superior orbit between the levator and the periorbita towards the supraorbital notch, where it exits accompanied by the supraorbital nerve. Other branches of the ophthalmic artery include the medial palpebral, supratrochlear, and dorsal nasal arteries. The infraorbital artery, a terminal branch of the maxillary artery, passes through the inferior orbital fissure into the infraorbital sulcus and gives off branches to the orbital fat and muscular branches to the inferior rectus and inferior oblique muscles before traversing the infraorbital canal to exit at the infraorbital foramen. It anastomoses with the angular artery and the inferior palpebral vessels.

\section{The venous drainage of the orbit}

The venous drainage of the orbit is through valveless superior and inferior ophthalmic veins that drain in a variable fashion. Unlike the arteries, the veins lie within the connective tissue septa of the orbit. The superior ophthalmic vein, the larger of the two, forms superomedially near the trochlea by the confluence of the angular, supraorbital, and supratrochlear veins. It is divided into three parts. The first part passes along the medial border of the superior rectus muscle. The second part passes posterolaterally under the superior rectus to enter the muscle cone. The third part passes posteriorly along the lateral border of the superior rectus to enter the superior orbital fissure, outside the annulus of Zinn, and drains into the cavernous sinus. The inferior ophthalmic vein is more variable and usually forms anteriorly as a plexus within the inferomedial orbital fat. It courses posteriorly along the inferior rectus and usually drains into the superior ophthalmic vein. It also communicates with the pterygoid plexus via the inferior orbital fissure. The ophthalmic veins receive tributaries from muscular, vortex, and medial and lateral collateral veins.

\section{The orbital fat and periorbita}

The globe and the other orbital tissue structures are surrounded by orbital fat which cushions the globe and 
aids movement. Anteriorly, the fat is dense and is divided into distinct compartments by a complex system of connective tissue septa characterized by Koorneef ${ }^{17,18}$ after studying thick serial sections of the orbit. Orbital movement is facilitated by sliding of the orbital septa over each other and motility restriction results from scarring of the orbital septa owing to trauma or inflammation. The posterior orbital septa are more diaphanous and the fat forms larger globules of decreased density.

The orbit is lined by a continuous fibrous membrane, the periorbita, which forms a dense condensation at the orbital margin. The latter, known as the arcus marginalis, gives rise to the orbital septum. The periorbita is firmly attached to the bone at the orbital margin, the suture lines, the trochlear fossa, fissures, the lateral tubercle, the anterior lacrimal crest, and the perforating vascular foramina. Otherwise it is fairly loosely attached and is easily dissected to gain entry into the relatively bloodless subperiosteal space.

\section{References}

1 Rootman J, Stewart B, Goldberg RA. Orbital Surgery: A Conceptual Approach. Lippincott - Raven: Philadelphia and New York, 1995, pp 79-146.

2 Kikkawa DO, Lemke BN. Orbital and eyelid anatomy. In: Dortzbach RK (ed). Ophthalmic Plastic Surgery: Prevention and Management of Complications. Raven Press: New York, 1994, pp 1-29.

3 Rene C, Rose GE, Lenthall R, Moseley I. Major orbital complications of endoscopic sinus surgery. $\mathrm{Br}$ J Ophthalmol 2001; 85: 598-603.
4 Clauser L, Carinci F, Galie M. Neurofibromatosis of the orbit and skull base. J Craniofac Surg 1998; 9: 280-284.

5 Davis S, King J. A case of pulsating proptosis. Med J Aust 1979; 1: 240-242.

6 Simonton JT, Garber PF, Ahl N. Margins of safety in lateral orbitotomy. Arch Ophthalmol 1977; 95: 1229-1231.

7 Yeh S, Foroozan R. Orbital apex syndrome. Curr Opin Ophthalmol 2004; 15: 490-498.

8 Onodi A. The optic nerve and the accessory sinuses of the nose: a contribution of canalicular neuritis and atrophy of the optic nerve of nasal origin. Ann Otol Rhinol Laryngol 1908; 17: 1-116.

9 Kainz J, Stammberger H. Danger areas of the posterior rhinobasis: an endoscopic and anatomical study. Acta Oto-Laryngol 1992; 112: 852-861.

10 Anderson RL, Panje WR, Gross CE. Optic nerve blindness following blunt forehead trauma. Ophthalmology 1982; 89: 445-455.

11 Sarkies N. Traumatic optic neuropathy. Eye 2004; 18: 1122-1125.

12 Segrest D, Dortzbach RK. Medial orbital wall fractures: complications and management. Ophthalmic Plast Reconstr Surg 1989; 5: 75-80.

13 Hayreh SS. Arteries of the orbit in the human being. $\mathrm{Br} J$ Surg 1963; 50: 938-953.

14 Hayreh SS. The ophthalmic artery. III: branches. Br J Ophthalmol 1962; 46: 212-247.

15 Hayreh SS, Dass R. The ophthalmic artery. II: intra-orbital course. Br J Ophthalmol 1962; 46: 165-185.

16 Hayreh SS, Dass R. The ophthalmic artery. I: origin and intra-cranial and intra-canalicular course. $\mathrm{Br} J$ Ophthalmol 1962; 46: 65-98.

17 Koorneef L. New insights in the human orbital connective tissue: results of a new anatomic approach. Arch Ophthalmol 1977; 95: 1269-1273.

18 Koorneef L. Orbital septa: anatomy and function. Ophthalmology 1979; 86: 876-885. 